\title{
Renormalization of parton quasi-distributions beyond the leading order: spacelike vs. timelike
}

\author{
V.M. Braun, ${ }^{a}$ K.G. Chetyrkin ${ }^{b, c}$ and B.A. Kniehl ${ }^{c}$ \\ ${ }^{a}$ Institut für Theoretische Physik, Universität Regensburg, \\ 93040 Regensburg, Germany \\ ${ }^{b}$ Institut für Theoretische Teilchenphysik, Karlsruhe Institute of Technology (KIT), \\ Wolfgang-Gaede-Straße 1, 76131 Karlsruhe, Germany \\ ${ }^{c}$ II. Institut für Theoretische Physik, Universität Hamburg, \\ Luruper Chaussee 149, 22761 Hamburg, Germany \\ E-mail: vladimir.braun@ur.de, Konstantin.Chetyrkin@kit.edu, \\ kniehl@desy.de
}

ABSTRACT: We argue that the renormalization factors for non-local quark-antiquark and gluon operators at space-like and time-like separations connected by a Wilson line coincide to all orders in perturbation theory. We calculate the anomalous dimensions and renormalization constants of quark-antiquark and gluon operators to three- and two-loop accuracy, respectively, and also compute vacuum expectation values of these operators to three-loop accuracy.

Keywords: NLO Computations, QCD Phenomenology

ARXIV EPRINT: 2004.01043 


\section{Contents}

1 Introduction 1

$\begin{array}{llr}2 & \text { Effective field theory } & 3\end{array}$

3 Calculation 5

3.1 Generalities 5

3.2 Renormalization constants and VEVs of qPDF operators 6

3.3 Reduction 9

3.4 Master integrals: time-like versus space-like 9

4 Transition to position space 11

5 Results $\quad 12$

$\begin{array}{ll}5.1 \text { Anomalous dimensions and renormalization constants } & 12\end{array}$

$\begin{array}{ll}5.2 \text { Correlation functions: momentum space } & 12\end{array}$

$\begin{array}{lll}5.3 \text { Correlation functions: position space } & 16\end{array}$

$\begin{array}{llr}6 & \text { Conclusions } & 17\end{array}$

$\begin{array}{lr}\text { A Anomalous dimensions } & 18\end{array}$

$\begin{array}{ll}\text { B Renormalization constants } & 19\end{array}$

\section{Introduction}

Studies of non-local gauge-invariant operators containing segments of Wilson lines have a long history. They emerged in connection with the attempts to reformulate gauge theories in terms of path-ordered gauge factors (Wilson lines), in particular within the loopspace formalism by Makeenko and Migdal [1]. The study of the renormalization of Wilson lines has been initiated by Polyakov [2], Gerwais and Neveau [3], and continued by several authors [4-7]; see ref. [8] for a review. The one-dimensional auxiliary-field formalism introduced in this context in refs. [3, 6] enables the application of the usual language of correlation functions of local operators and is important both conceptually and at a technical level, as a basis for multiloop calculations. Subsequent applications of these methods have been to the study of infrared singularities in Feynman amplitudes, starting from the work of refs. [9, 10], heavy-quark effective theory (HQET) [11-15] and transverse-momentumdependent (TMD) factorization [16]. 
Recently, there has been renewed interest in the study of matrix elements of non-local off-light-cone operators of the type

$$
\begin{aligned}
\mathbb{Q}(z) & =\bar{q}(z v) \Gamma[z v, 0] q(0), \\
\mathbb{G}_{\mu \nu \alpha \beta}(z) & =g^{2} F_{\mu \nu}(z v)[z v, 0] F_{\alpha \beta}(0),
\end{aligned}
$$

where $q(x)$ is a quark field, $F_{\mu \nu}(x)$ is the gluon field strength tensor, $\Gamma$ is a certain Dirac structure, $v^{\mu}$ is an auxiliary four-vector and $z$ is a real number. In addition, $[z v, 0]$ is a straight-line-ordered Wilson line connecting the two fields,

$$
[z v, 0]=\mathcal{P} \exp \left[i g \int_{0}^{z} d z^{\prime} v^{\mu} A_{\mu}\left(z^{\prime} v\right)\right]
$$

which we assume to be taken in the proper representation of the gauge group, fundamental for quarks and adjoint for gluons. Matrix elements of such operators acting on hadron states with large momenta are often referred to as parton quasi-distributions (qPDFs) [17] or pseudo-distributions [18]. They can be factorized in terms of parton distribution functions (PDFs) [19] and, at the same time, are accessible in lattice calculations if the quarkantiquark separation is chosen to be space-like, $v^{2} \equiv v^{\mu} v_{\mu}<0$. It was suggested that PDFs can be constrained in this way [17], and this possibility is being intensively explored, see, e.g., refs. [20, 21] for reviews. The rationale for using matrix elements of the operators in eqs. (1.1) and (1.2) in these studies is that they are "cheaper" to compute on the lattice as compared to other Euclidean observables with similar factorization properties; see, e.g., refs. [22-24]. This advantage comes at the price that the renormalization of the non-local operators in eqs. (1.1) and (1.2) is nontrivial and requires special attention [19, 25-33].

In this paper, we address the question whether computational methods familiar from HQET can be applied to the calculation of the renormalization constants (RCs) of the operators in eq. (1.1), alias for qPDFs, in high orders. The difference is that, in HQET, the heavy-quark velocity $v^{\mu}$ is time-like, $v^{2}>0$, while, for qPDF studies, it is spacelike, $v^{2}<0$. We argue that the change of sign has no effect on the renormalization and confirm this result by an explicit calculation to three-loop accuracy. This result is also relevant in the context of TMD factorization, where Wilson lines are shifted off the light cone to regularize rapidity divergences in TMD operators [16]. Our statement is that the anomalous dimensions (ADs) and RCs do not depend on the direction, space-like or time-like. To avoid confusion, in this work, we imply using dimensional regularization. In renormalization schemes with an explicit regularization scale, the Wilson line in eq. (1.3) suffers from an additional linear ultraviolet divergence [2], which has to be removed. This can be done by mass renormalization, similarly to the introduction of the residual mass term in HQET, or, alternatively, by considering a suitable ratio of matrix elements involving the same operator [34, 35]. Having in mind the second approach, we calculate in this work the vacuum expectation values (VEVs) of the operators in eqs. (1.1) and (1.2) to three-loop accuracy for space-like and time-like choices of the auxiliary vector $v^{\mu}$. VEVs of non-local gluon operators are also of interest for studies of the QCD vacuum structure; see ref. [36] for a review and further references. The gluon case is also interesting as the generic gluon 
operator as defined in eq. (1.2) is not renormalized multiplicatively. The calculation of its VEV allows one to obtain the renormalization constants avoiding the necessity to consider mixing with non-gauge-invariant operators. In this way, we verify the mixing pattern found in refs. [37, 38] and also calculate the two-loop mixing matrix, which is another new result.

This paper is organized as follows. In section 2, we recall the standard argument that any Green function in QCD with the insertion of the non-local operator in eq. (1.1) may be obtained from the correlation function of two appropriate local "heavy-light" operators and verify this diagrammatically. In section 3, we introduce the formalism to be used here, explain the reduction of Feynman diagrams to master integrals and discuss the relation of Green functions between the time-like and space-like regions. In section 4, we explain how the relationship between time-like and space-like Green functions translates from momentum space to position space. In section 5 , we present our analytic and numerical results for the correlators of interest here, both in momentum and position space. Section 6 contains our conclusions. For the reader's convenience, we list the analytic results through three loops for the ADs and RCs entering our analysis in appendices A and B, respectively.

\section{Effective field theory}

As is well known, the interaction of a particle propagating along a classical path in the background gauge field reduces to the path-ordered phase factor along its trajectory. Such an auxiliary classical particle can be simulated by supplementing the QCD Lagrangian $\mathcal{L}_{\mathrm{QCD}}$ by an extra term,

$$
\mathcal{L}=\mathcal{L}_{\mathrm{QCD}}+\bar{h}_{v} \mathrm{i} v^{\mu} \mathcal{D}_{\mu} h_{v}
$$

where $h_{v}$ is a (complex) scalar field in either fundamental or adjoint representation of the gauge group, $\mathcal{D}=\partial_{\mu}-i g A_{\mu}^{a} T^{a}$ is the covariant derivative and $T^{a}$ are the $\mathrm{SU}(3)$ generators in the appropriate representation. The Lagrangian in eq. (2.1) for $v^{2}>0$ is, essentially, the standard HQET Lagrangian [39], apart from our choice of $h_{v}(x)$ as a scalar. The case $v^{2}<0$ is of interest in connection with qPDFs [27,31]. Without loss of generality, we can assume $v_{0}>0$ and the usual causal boundary conditions for the "heavy" field $h_{v}$. Its free propagator reads

$$
\begin{aligned}
S_{h}^{(0)}(x) \equiv\left\langle 0\left|\mathrm{~T}\left\{h_{v}^{i}(x) \bar{h}_{v}^{j}(0)\right\}\right| 0\right\rangle & =\delta^{i j} \int \frac{d^{D} k}{i(2 \pi)^{D}} e^{-i k \cdot x} \frac{1}{-v \cdot k-i \epsilon} \\
& =\delta^{i j} \int_{0}^{\infty} d s \delta^{(D)}(x-s v) \\
& =\delta^{i j} \frac{1}{|v|} \theta\left(\frac{v \cdot x}{v^{2}}\right) \delta^{(D-1)}\left(x_{\perp}\right),
\end{aligned}
$$

where $x_{\perp}^{\mu}=x^{\mu}-v^{\mu}(v \cdot x) / v^{2}$ and $i, j$ are color indices. Adding the interactions with the gluon field, one obtains $[8]^{1}$

$$
\left\langle 0\left|\mathrm{~T}\left\{h_{v}^{i}(x) \bar{h}_{v}^{j}(0)\right\}\right| 0\right\rangle_{A}=S_{h}^{(0)}(x)[x, 0],
$$

\footnotetext{
${ }^{1}$ Notice that the straight-line-ordered Wilson line $[x, 0]$ is the unique solution of the differential equation $(x \cdot D)[x, 0]=0$ with the boundary condition $[0,0]=1$, whereas the propagator of the "heavy" field is a Green function of the same operator. Thus they differ by a factor which is just the free propagator.
} 

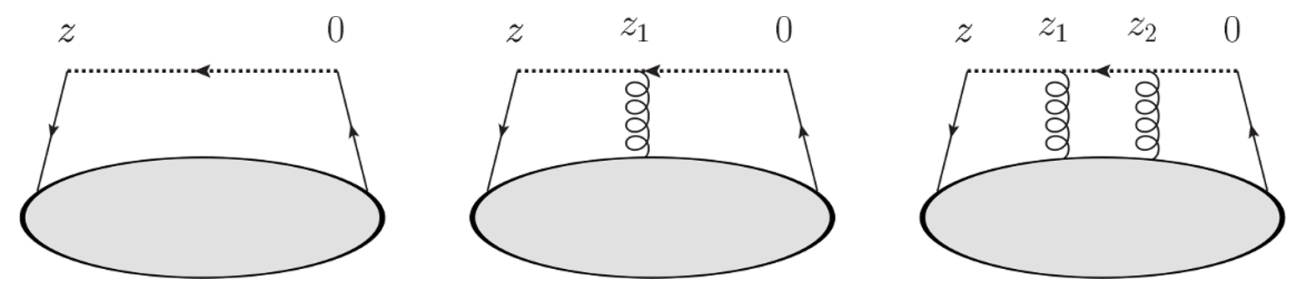

Figure 1. The first three "generic" diagrams for an insertion of the operator $\mathbb{Q}(z)$ in eq. (1.1). Wilson lines are indicated as dotted.

so that any Green function in QCD with the insertion of the non-local operator in eq. (1.1) can equivalently be obtained from the correlation function of two local "heavy-light" operators. E.g. for quarks,

$$
S_{h}^{(0)}(v z)\left\langle 0\left|\mathrm{~T}\left\{O(z) \Phi\left(x_{1}, \ldots, x_{n}\right)\right\}\right| 0\right\rangle=\left\langle 0\left|\mathrm{~T}\left\{\left(\bar{q} h_{v}\right)(z v) \Gamma\left(\bar{h}_{v} q\right)(0) \Phi\left(x_{1}, \ldots, x_{n}\right)\right\}\right| 0\right\rangle,
$$

where $\Phi\left(x_{1}, \ldots, x_{n}\right)$ stands for an arbitrary set of QCD fields at positions $x_{1}, \ldots, x_{n}$, and similarly for gluons. With our choice $v_{0}>0$ and for $z>0$, the path ordering in the operator on the l.h.s. of eq. (2.4) is consistent with time ordering. For $z<0$, the expressions on the 1.h.s. and r.h.s. of eq. (2.4) both vanish. It is instructive to verify the equivalence in eq. (2.4) diagrammatically at the few lowest orders in perturbation theory. For definiteness, consider quark operators. On the one hand, from the definition of the path-ordered exponential in eq. (1.3), one obtains

$$
\mathbb{Q}(z)=\bar{q}(z v) \Gamma\left[1+i g \int_{0}^{z} d z_{1} A_{v}\left(z_{1} v\right)+(i g)^{2} \int_{0}^{z} d z_{1} \int_{0}^{z_{1}} d z_{2} A_{v}\left(z_{1} v\right) A_{v}\left(z_{2} v\right)+\cdots\right] q(0)
$$

where we have used the shorthand notation $A_{v}=v^{\mu} A_{\mu}$. Notice that the factor $1 / 2$ ! that naively appears in the third term of the expansion of the exponential function is replaced by the ordering of the integration regions. On the other hand, one can easily write the corresponding expression using the Feynman rules of the effective theory in eq. (2.1),

$$
\begin{gathered}
\mathrm{T}\left\{\left(\bar{q} h_{v}\right)(z v) \Gamma\left(\bar{h}_{v} q\right)(0)\right\}=\frac{1}{|v|} \theta(z) \delta^{(D-1)}\left(0_{\perp}\right) \bar{q}(z v) \Gamma\left[1+i g \int_{0}^{\infty} d z_{1} \theta\left(z-z_{1}\right) A_{v}\left(z_{1} v\right)\right. \\
\left.+(i g)^{2} \int_{0}^{\infty} d z_{2} \int_{0}^{\infty} d z_{1} \theta\left(z-z_{1}-z_{2}\right) A_{v}\left(z_{1} v+z_{2} v\right) A_{v}\left(z_{2} v\right)+\ldots\right] q(0) .
\end{gathered}
$$

In this way, the $1 / 2$ ! factor in the last term is compensated by a different mechanism: there are two equivalent ways to couple the four $h_{v}$ fields in the Lagrangian insertions $(i g)^{2} / 2 !\left(\bar{h}_{v} A_{v}^{a} T_{a} h_{v}\right)^{2}$ with those in the currents $\bar{q} h_{v}(z v)$ and $\bar{h}_{v} q(0)$. Notice that disconnected diagrams are not counted. The two expressions in eqs. (2.5) and (2.6) are obviously equal up to an overall factor, which is nothing but the free propagator of the "heavy" field, $S_{h}^{(0)}(z v)$. 


\section{Calculation}

\subsection{Generalities}

We define the renormalization factors for generic composite operators $O$ as

$$
O(z)=Z_{O} O_{B}
$$

where $O_{B}$ is the corresponding bare operator and the bare fields are related to the renormalized ones as

$$
A_{\mu}^{a, B}=\sqrt{Z_{3}} A_{\mu}^{a}, \quad q_{B}=\sqrt{Z_{2}} q, \quad h_{v}^{B}=\sqrt{Z_{h}} h_{v} .
$$

The bare QCD coupling constant $g_{B}$ is expressed as

$$
g_{B}=Z_{g} \mu^{\epsilon} g,
$$

where $\mu$ is the 't Hooft mass and $\epsilon=(4-D) / 2$, with $D$ being the running space-time dimension within the method of dimensional regularization [40-42]. Within the modified minimal-subtraction $(\overline{\mathrm{MS}})$ scheme, every $\mathrm{RC}$ is independent of dimensional parameters (masses and momenta) and can be represented as

$$
Z(a)=1+\sum_{n=1}^{\infty} \frac{z^{(n)}(a)}{\epsilon^{n}}
$$

where $a=g^{2} /\left(16 \pi^{2}\right)$. Given a RC $Z(a)$, the corresponding AD is defined as

$$
\gamma(a)= \pm \mu^{2} \frac{d \ln Z(a)}{d \mu^{2}}= \pm\left(-a \frac{\partial z^{(1)}(a)}{\partial a}\right)=\sum_{n=1}^{\infty}(\gamma)_{n} a^{n} .
$$

Here, the sign depends on the way how the considered bare quantity, $O_{B}$, is related to its renormalized counterpart, $O$. With our convention (3.1), the renormalization group (RG) equation for $O$ assumes the form

$$
\mu^{2} \frac{d O}{d \mu^{2}}=\gamma_{O} O \quad \text { with } \quad \gamma_{O}=\mu^{2} \frac{d \ln Z_{O}(a)}{d \mu^{2}} .
$$

Traditionally, in renormalizing coupling constants, wave functions and masses, the defining relation (3.1) is written with $Z_{O}$ on the left-hand side, e.g.,

$$
Z_{m} m=m_{B}
$$

As a result, the ADs $\gamma_{2}$ and $\gamma_{h}$, and the $\beta$ function (see below) are defined with the minus sign version of eq. (3.5), but the ADs of composite operators (see below) with the plus sign. Customarily, one also defines $Z_{a}=Z_{g}^{2}$ and refers to the corresponding $\mathrm{AD}$ as the $\mathrm{QCD} \beta$ function,

$$
\beta(a)=a \frac{\partial z_{a}^{(1)}(a)}{\partial a}=\sum_{n=1}^{\infty} \beta_{n} a^{n} .
$$


The RCs $Z_{2}$ and $Z_{a}$ serve to renormalize the standard QCD Lagrangian and have been well known through three loops for a long time [43-45]. The RC $Z_{h}$ is known to the same accuracy for the time-like case $v^{2}>0$ [13] and will be used by us as convenient reference point. For the reader's convenience, we quote the RCs as well as the corresponding ADs in appendices B and A, respectively. Due to an overall $\delta^{(D-1)}\left(x_{\perp}\right)$ factor in position space, the self-energy and hence also the propagator of the "heavy" field $h_{v}$ can only depend on the projection of its momentum onto $v^{\mu}$, for which we use the notation

$$
\omega=p \cdot v .
$$

The heavy-field self-energy is defined, as usual, as the sum of one-particle-irreducible amputated diagrams,

$$
\omega \Sigma_{h}(\omega)=i \int d^{D} x e^{i p \cdot x}\left\langle 0\left|T\left\{h_{v}(x) \bar{h}_{v}(0)\right\}\right| 0\right\rangle^{1 \mathrm{PI}, \text { amputated }},
$$

and the full propagator is then given by

$$
\tilde{S}_{h}(\omega)=\frac{\tilde{S}_{h}^{0}(\omega)}{1+\Sigma_{h}(\omega)}, \quad \tilde{S}_{h}^{0}(\omega)=\frac{1}{-\omega-i 0} .
$$

Notice that the full propagator and the self-energy in Landau gauge satisfy the RG equations,

$$
\begin{aligned}
& \left(\mu^{2} \frac{\partial}{\partial \mu^{2}}+\beta(a) a \frac{\partial}{\partial a}\right) \tilde{S}_{h}=\gamma_{h} \tilde{S}_{h} \\
& \left(\mu^{2} \frac{\partial}{\partial \mu^{2}}+\beta(a) a \frac{\partial}{\partial a}\right) \Sigma_{h}=-\gamma_{h} \Sigma_{h} .
\end{aligned}
$$

The renormalization factors $Z_{Q}$ of the "heavy-light" operators in eq. (3.13) are calculated from the corresponding vertex functions $\Gamma(p, \omega)$ as explained, e.g., in ref. [46]; the general formalism is the same for $v^{2}>0$ and $v^{2}<0$.

\subsection{Renormalization constants and VEVs of qPDF operators}

Let us first consider quark operators. Let

$$
Q_{i}(x)=\bar{q}_{i}(x) h_{v}(x)
$$

where $i$ is the spinor index, which we do not show in what follows. One can show that this operator is multiplicatively renormalized. The corresponding $\mathrm{RC} Z_{Q}$,

$$
Q(x)=Z_{Q} Q_{B}(x)
$$

was calculated to three-loop accuracy for the time-like case $v^{2}>0$ [13] and is the same for space-like $v^{2}<0$. The RC $Z_{\mathbb{Q}}$ of the non-local operator defined in eq. (1.1) is related to $Z_{Q}$ as $[27]$

$$
\mathbb{Q}(z)=Z_{\mathbb{Q}} \mathbb{Q}_{B}(z), \quad Z_{\mathbb{Q}}=Z_{Q}^{2}
$$


The corresponding ADs are obviously related as

$$
\gamma_{\mathbb{Q}}(z)=2 \gamma_{Q} .
$$

The equality (3.15) implies that $Z_{\mathbb{Q}}$ does not depend on the Dirac structure $\Gamma$ in the definition of the operator $\mathbb{Q}(z)$, eq. (1.1). We have checked this relation at the two-loop level by explicit calculation of the VEV of $\mathbb{Q}(z)$ to three-loop accuracy; see section 5 . This VEV is non-vanishing in perturbation theory only for the Dirac structure $\Gamma=\gamma_{\mu}$, in which case it follows from Lorentz invariance that $\left\langle 0\left|\mathbb{Q}_{\mu}\right| 0\right\rangle \propto v_{\mu}$. Thus, it is sufficient to consider

$$
\Pi(z)=\left\langle 0\left|v^{\mu} \mathbb{Q}_{\mu}(z)\right| 0\right\rangle=\langle 0|\bar{q}(z v) \psi[z v, 0] q(0)| 0\rangle,
$$

or, equivalently, the momentum space correlation function,

$$
\widetilde{\Pi}(\omega)=i \int d^{D} x e^{i p \cdot x}\left\langle 0 \mid \mathrm{T}\left\{Q(x) \psi Q^{\dagger}(0)\right\}\right\rangle,
$$

where the current $Q(x)$ is defined in eq. (3.13). It is easy to see that

$$
\Pi(z>0)=\frac{1}{i} \int d^{D-1} x_{\perp} \int \frac{d^{D} p}{(2 \pi)^{D}} e^{-i p \cdot x} \tilde{\Pi}(p) \quad \text { with } \quad z= \pm x \cdot v,
$$

where the $\pm \operatorname{sign}$ corresponds to the choice $v^{2}= \pm 1$. Notice that $\Pi(z>0)$ does not depend on the "contact" terms in $\tilde{\Pi}(p)$ of the form const $\cdot \omega^{2}$, which suffer from an extra UV divergence coming from the integration region around $x=0$ in eq. (3.18). Thus, in momentum space, it is natural to consider instead of $\tilde{\Pi}(p)$ an analog of the Adler function, namely

$$
\tilde{D}(\omega)=\omega \frac{d}{d \omega}\left(\frac{\pi^{2}}{\omega^{2}} \tilde{\Pi}(\omega)\right)
$$

The corresponding RG equation for $D(\omega)$ reads

$$
\left(\mu^{2} \frac{\partial}{\partial \mu^{2}}+\beta(a) a \frac{\partial}{\partial a}\right) \tilde{D}=2 \gamma_{Q} \tilde{D} .
$$

The gluon case is more involved because, in the presence of the external four-vector $v^{\mu}$, the components parallel and transverse to $v^{\mu}$ can be renormalized differently. Introducing the corresponding projection operators,

$$
g_{\mu \nu}^{\|}=\frac{v_{\mu} v_{\nu}}{v^{2}}, \quad g_{\mu \nu}^{\perp}=g_{\mu \nu}-\frac{v_{\mu} v_{\nu}}{v^{2}},
$$

we can define two multiplicatively renormalizable gauge-invariant local operators in effective theory with adjoint "heavy" scalars as ${ }^{2}$

$$
\begin{aligned}
G_{\mu \nu}^{\| \perp}(x) & =\left[g_{\mu \alpha}^{\|} g_{\nu \beta}^{\perp}-g_{\nu \alpha}^{\|} g_{\mu \beta}^{\perp}\right] g F^{\alpha \beta}(x) h_{v}(x), \\
G_{\mu \nu}^{\perp \perp}(x) & =g_{\mu \alpha}^{\perp} g_{\nu \beta}^{\perp} g F^{\alpha \beta}(x) h_{v}(x),
\end{aligned}
$$

\footnotetext{
${ }^{2}$ Notice that we include the QCD coupling $g$ in the definition of the operators, which simplifies the renormalization factors.
} 
with the RCs $Z_{\| \perp}$ and $Z_{\perp \perp}$,

$$
G_{\mu \nu}^{\| \perp}=Z_{\| \perp}\left(G_{\mu \nu}^{\| \perp}\right)_{B}, \quad G_{\mu \nu}^{\perp \perp}=Z_{\perp \perp}\left(G_{\mu \nu}^{\perp \perp}\right)_{B} .
$$

We will denote the corresponding ADs as $\gamma_{\| \perp}$ and $\gamma_{\perp \perp}$ respectively. The correlation functions of these operators have the form

$$
\begin{aligned}
\left\langle 0\left|G_{\mu \nu}^{\perp \perp}(x) \bar{G}_{\alpha \beta}^{\perp \perp}(0)\right| 0\right\rangle & =\left(g_{\mu \alpha}^{\perp} g_{\nu \beta}^{\perp}-g_{\nu \alpha}^{\perp} g_{\mu \beta}^{\perp}\right) \Pi_{\perp \perp}(x), \\
\left\langle 0\left|G_{\mu \nu}^{\| \perp}(x) \bar{G}_{\alpha \beta}^{\| \perp}(0)\right| 0\right\rangle & =\left(g_{\mu \alpha}^{\|} g_{\nu \beta}^{\perp}-g_{\nu \alpha}^{\|} g_{\mu \beta}^{\perp}-g_{\mu \beta}^{\|} g_{\nu \alpha}^{\perp}+g_{\nu \beta}^{\|} g_{\mu \alpha}^{\perp}\right) \Pi_{\| \perp}(x), \\
\left\langle 0\left|G_{\mu \nu}^{\perp \perp}(x) \bar{G}_{\alpha \beta}^{\| \perp}(0)\right| 0\right\rangle & =0,
\end{aligned}
$$

where $\bar{G}_{\alpha \bar{\beta}}^{\perp \perp}=g_{\mu \alpha}^{\perp} g_{\nu \beta}^{\perp} g F^{\alpha \beta} \bar{h}_{v}$, etc., and are renormalized by squares of the corresponding RCs in eq. (3.24). In this notation, a generic two-gluon vacuum correlation function related to the qPDF operator in eq. (1.2) takes the form

$$
\begin{aligned}
\Pi_{\mu \nu \alpha \beta}(x)= & \left\langle 0\left|g^{2} F_{\mu \nu}(x) h_{v}(x) \bar{h}_{v}(0) F_{\alpha \beta}(0)\right| 0\right\rangle \\
= & \left(g_{\mu \alpha}^{\perp} g_{\nu \beta}^{\perp}-g_{\nu \alpha}^{\perp} g_{\mu \beta}^{\perp}\right) \Pi_{\perp \perp}(x)+\left(g_{\mu \alpha}^{\|} g_{\nu \beta}^{\perp}-g_{\nu \alpha}^{\|} g_{\mu \beta}^{\perp}-g_{\mu \beta}^{\|} g_{\nu \alpha}^{\perp}+g_{\nu \beta}^{\|} g_{\mu \alpha}^{\perp}\right) \Pi_{\| \perp}(x) \\
= & \left(g_{\mu \alpha} g_{\nu \beta}-g_{\nu \alpha} g_{\mu \beta}\right) \Pi_{\perp \perp}(x) \\
& +\frac{1}{v^{2}}\left(v_{\mu} v_{\alpha} g_{\nu \beta}-v_{\nu} v_{\alpha} g_{\mu \beta}-v_{\mu} v_{\beta} g_{\nu \alpha}+v_{\nu} v_{\beta} g_{\mu \alpha}\right)\left[\Pi_{\| \perp}(x)-\Pi_{\perp \perp}(x)\right] .
\end{aligned}
$$

For future reference, we introduce the corresponding "Adler" functions:

$$
\begin{aligned}
& \tilde{D}_{\perp \perp}(\omega)=\omega \frac{d}{d \omega}\left(\frac{\tilde{\Pi}_{\perp \perp}(\omega)}{\omega^{3}}\right), \\
& \tilde{D}_{\| \perp}(\omega)=\omega \frac{d}{d \omega}\left(\frac{\tilde{\Pi}_{\| \perp}(\omega)}{\omega^{3}}\right),
\end{aligned}
$$

where

$$
\widetilde{D}_{\perp \perp}(\omega)=i \int d^{D} x e^{i p \cdot x} \Pi_{\perp \perp}(x), \quad \widetilde{D}_{\| \perp}(\omega)=i \int d^{D} x e^{i p \cdot x} \Pi_{\| \perp}(x) .
$$

The functions $\tilde{D}_{\perp \perp}(\omega)$ and $\tilde{D}_{\| \perp}(\omega)$ satisfy the standard RG equations like eq. (3.21) with the ADs $\left(2 \gamma_{\perp \perp}\right)$ and $\left(2 \gamma_{\| \perp}\right)$ respectively. In the qPDF literature, following refs. [37, 38], one usually introduces a different operator basis,

$$
J_{1}^{\mu \nu}=g F^{\mu \nu} h_{v}, \quad J_{2}^{\mu \nu}=\frac{v_{\rho}}{v^{2}} g\left(F_{a}^{\mu \rho} v^{\nu}-F_{a}^{\nu \rho} v^{\mu}\right) h_{v}^{a},
$$

so that, obviously,

$$
J_{2}^{\mu \nu}(x)=G_{\mu \nu}^{\| \perp}(x), \quad J_{1}^{\mu \nu}(x)=G_{\mu \nu}^{\perp \perp}(x)+G_{\mu \nu}^{\| \perp}(x) .
$$

The operators $J_{1}$ and $J_{2}$ are renormalized by a triangular $2 \times 2$ mixing matrix $Z_{i k}$ such that $Z_{21}=0$ and $Z_{12}-Z_{22}+Z_{11}=0$ to all orders. These relations imply that

$$
Z_{11}=Z_{\perp \perp}, \quad Z_{22}=Z_{\| \perp} .
$$

Notice that we ignore mixing with gauge-noninvariant operators as they do not contribute to gauge-invariant observables. With the main definitions at hand, we proceed to describe the calculation procedure. 


\subsection{Reduction}

The generation of the Feynman diagrams and their reduction to master integrals (MIs) have been done in the standard way, using the programs QGRAF [47] and FIRE6 [48], respectively. ${ }^{3}$

The reduction typically results in a sum of MIs with coefficients being rational functions of the space-time dimension $D$. In addition, every MI is multiplied by a factor of the form

$$
\left(v^{2}\right)^{n_{1}} \omega^{n_{2}},
$$

and color factors like $C_{F}, C_{A}$, etc. We have checked that our results for the time-like case $v^{2}=1$ are in full agreement with ref. [13]. In our calculation, we have used the values of the relevant MIs given in ref. [13].

\subsection{Master integrals: time-like versus space-like}

The MIs for $v^{2}>0$, which we refer to as time-like, are analytic functions of $\omega$. They are real for $\omega<0$ and have a branch cut at $\omega>0$. On dimensional grounds, each MI has the form

$$
M\left(\omega<0, v^{2}=1 ; \mathbf{n}\right)=(-2 \omega)^{d^{M}} M(\epsilon ; \mathbf{n}), \quad d^{M}=D L-2 \sum_{l} n_{l}-\sum_{h} n_{h},
$$

where $M(\epsilon ; \mathbf{n})$ is a real function of the space-time dimension $D, d^{M}$ is the dimension of the MI $M, L$ stands for the number of loops and the sums over $l$ and $h$ count the indices $n_{l}$ and $n_{h}$ of all usual QCD ("light") and special ("heavy") lines of the integral. The argument $\mathbf{n}$ stands for the collection of all indices. We assume that every MI is of scalar type, so that the corresponding integrand is given by a product of denominators involving "light" (massless) and "heavy" propagators, possibly raised to certain (integer) powers (indices). The reduction to scalar MIs is certainly possible at the three-loop level; see, e.g., refs. $[13,51]$. The restriction to the normalization $v^{2}=1$ can easily be relaxed. Indeed, the "heavy" propagator in eq. (2.2) is a homogeneous function w.r.t. the rescaling $v^{\mu} \rightarrow \lambda v^{\mu}$. Thus, we have $M\left(\lambda \omega, \lambda^{2} v^{2} ; \mathbf{n}\right)=\lambda^{-d_{v}^{M}} M\left(\omega, v^{2} ; \mathbf{n}\right)$, where $d_{v}^{M}=-\sum_{h} n_{h}$ stands for the $v$ dimension of the integral, so that, for generic time-like $v^{2}>0$, we have

$$
\begin{aligned}
M\left(\omega<0, v^{2}>0 ; \mathbf{n}\right) & =(-2 \omega)^{d^{M}}\left(v^{2}\right)^{\left(-d^{M}+d_{v}^{M}\right) / 2} M(\epsilon ; \mathbf{n}) \\
& =(-2 \omega)^{d_{v}^{M}}\left(\frac{4 \omega^{2}}{v^{2}}\right)^{\left(d^{M}-d_{v}^{M}\right) / 2} M(\epsilon ; \mathbf{n}) .
\end{aligned}
$$

The result for $\omega>0$ is obtained by analytic continuation. In this way, the MI acquires an imaginary part according to the usual causal prescription $\omega \rightarrow \omega+i 0$, so that $4 \omega^{2} \rightarrow$ $(-2 \omega-i 0)^{2}$. In order to calculate a MI for the space-like case $v^{2}<0$, it is useful to start from the so-called $\alpha$ representation of the time-like MI, ${ }^{4}$

$$
M\left(\omega, v^{2}>0 ; \mathbf{n}\right)=\frac{\Gamma(\Sigma n-L d / 2)}{\prod_{\alpha} \Gamma\left(n_{\alpha}\right)} \int \prod_{\alpha} d z_{\alpha} z_{\alpha}^{n_{\alpha}-1} \delta(1-\Sigma z) \frac{(F-i 0)^{L d / 2-\Sigma n}}{U^{(L+1) d / 2-\Sigma n}},
$$

\footnotetext{
${ }^{3}$ We have also used the Mathematica program LiteRed 1.4 [49, 50] and the REDUCE package Grinder [51] for testing purposes and the identification of the MIs.

${ }^{4}$ Within HQET, this was considered in ref. [52]. A general discussion can be found, e.g., in refs. [50, 53].
} 
where $\Sigma n=\sum_{\alpha} n_{\alpha} \equiv \sum_{l} n_{l}+\sum_{h} n_{h}, \Sigma z=\sum_{\alpha} z_{\alpha}$, and $U$ and $F$ are homogeneous polynomials of degree $L$ and $L+1$ in the integration parameters $z_{\alpha}$, respectively. The function $U$ does not depend on kinematic invariants, whereas the function $F$ can be written as

$$
F=-2 \omega T_{p}+v^{2} T_{v},
$$

with polynomials $T_{p}$ and $T_{v}$ that only depend on the parameters $z_{\alpha}$ and are defined to be positive in the integration region of eq. (3.35). Notice that, in eq. (3.35), we do not assume that $\omega<0$, so that, for $v^{2}>0$, the MI acquires an imaginary part at $\omega>0$ according to the Feynman prescription $F \mapsto F-i 0$. The crucial observation is that, if one simultaneously changes the signs of $v^{2}$ and $\omega$, the MI receives an overall phase factor,

$$
\begin{aligned}
M\left(\omega>0, v^{2}<0 ; \mathbf{n}\right) & =e^{-i \pi(L d / 2-\Sigma n)} M\left(-\omega,\left|v^{2}\right| ; \mathbf{n}\right) \\
& =e^{i \pi \epsilon L}(-1)^{\Sigma n} M\left(-\omega,\left|v^{2}\right| ; \mathbf{n}\right),
\end{aligned}
$$

as, by definition,

$$
(F-i 0)^{\lambda}:= \begin{cases}F^{\lambda} & \text { if } F>0 \\ (-F)^{\lambda} e^{-i \pi \lambda} & \text { if } F<0 .\end{cases}
$$

Thus, we obtain

$$
\begin{aligned}
M\left(\omega>0, v^{2}<0 ; \mathbf{n}\right) & =e^{i \pi \epsilon L}(-1)^{\Sigma n}|2 \omega|^{d^{M}}\left|v^{2}\right|^{\left(-d^{M}+d_{v}^{M}\right) / 2} M(\epsilon ; \mathbf{n}) \\
& =e^{i \pi \epsilon L}(-1)^{\left(d_{0}^{M}+d_{v}^{M}\right) / 2}|2 \omega|^{d^{M}}\left|v^{2}\right|^{\left(-d^{M}+d_{v}^{M}\right) / 2} M(\epsilon ; \mathbf{n}) \\
& =(-2 \omega)^{d_{v}^{M}}\left(\frac{4 \omega^{2}}{v^{2}}-i 0\right) \stackrel{\left(d^{M}-d_{v}^{M}\right) / 2}{M(\epsilon ; \mathbf{n}),}
\end{aligned}
$$

where $d_{0}^{M}=\left.d^{M}\right|_{\epsilon=0}$. A generic Green function $G\left(\omega, v^{2}\right)$ with mass dimension $d^{G}$ and $v$ dimension $d_{v}^{G}$ is given by the sum of MIs multiplied by extra kinematic factors $\left(v^{2}\right)^{j_{1}}(-2 \omega)^{j_{2}}$, where $j_{1}$ and $j_{2}$ are integers that satisfy the obvious relations

$$
j_{2}+d^{M}=d^{G}, \quad 2 j_{1}+j_{2}+d_{v}^{M}=d_{v}^{G} .
$$

It is easy to see that

$$
\begin{aligned}
& \left(v^{2}\right)^{j_{1}}(-2 \omega)^{j_{2}} M\left(\omega<0, v^{2}>0 ; \mathbf{n}\right)=(-2 \omega)^{d_{v}^{G}}\left(\frac{4 \omega^{2}}{v^{2}}\right)^{\left(d^{G}-d_{v}^{G}\right) / 2} M(\epsilon ; \mathbf{n}), \\
& \left(v^{2}\right)^{j_{1}}(-2 \omega)^{j_{2}} M\left(\omega>0, v^{2}<0 ; \mathbf{n}\right)=(-2 \omega)^{d_{v}^{G}}\left(\frac{4 \omega^{2}}{v^{2}}-i 0\right)^{\left(d^{G}-d_{v}^{G}\right) / 2} M(\epsilon ; \mathbf{n}),
\end{aligned}
$$

so that, upon this multiplication, the mass and $v$ dimensions of a particular MI are substituted by those of the Green function in question and are the same for the contributions of all MIs and for all Feynman diagrams. As a consequence, going over from $v^{2}>0, \omega<0$ to $v^{2}<0, \omega>0$, the Green function acquires an overall phase factor,

$$
\begin{aligned}
G\left(\omega>0, v^{2}<0\right) & =e^{i \pi\left(d^{G}+d_{v}^{G}\right) / 2} G\left(-\omega,\left|v^{2}\right|\right) \\
& =e^{i \pi \epsilon L}(-1)^{\left(d_{0}^{G}+d_{v}^{G}\right) / 2} G\left(-\omega,\left|v^{2}\right|\right),
\end{aligned}
$$


where $d_{0}^{G}=\left.d^{G}\right|_{\epsilon=0}$, which is the final result. Thus, we conclude the following:

- A generic Green function at $v^{2}<0$ can be obtained from the result at $v^{2}>0$ by the (possible) global sign change $(-1)^{\left(d_{0}^{G}+d_{v}^{G}\right) / 2}$, which is the same to all orders of perturbation theory, and the formal substitution

$$
\left.(-2 \omega)^{d_{0}^{G}} \ln (-2 \omega-i 0)\right|_{v^{2}=1} \rightarrow(2 \omega)^{d_{0}^{G}}\left[\ln (2 \omega+i 0)-i \frac{\pi}{2}\right]_{v^{2}=-1},
$$

where we assume $\left|v^{2}\right|=1$ and the Feynman causal prescription.

- Since, in minimal schemes, the RCs and ADs neither depend on the global sign nor on the value of the external momentum, they are not affected by the analytic continuation in $v^{2}$ and are the same for time-like $\left(v^{2}=1\right)$ and space-like $\left(v^{2}=-1\right)$ kinematics.

\section{Transition to position space}

Without loss of generality, we may assume $v^{\mu}=(1,0,0,0)$ and $v^{\mu}=(0,0,0,1)$ for the timelike and space-like cases, respectively. For this choice, the variable $\omega$ is the energy, $\omega=p_{0}$, for the time-like case and the $z$ component of the momentum up to a minus sign, $\omega=-p_{z}$, for the space-like case. The relation between generic correlation functions established in the previous section, therefore, connects a $v^{2}=1$ Green function for negative energy with the corresponding $v^{2}=-1$ Green function with negative momentum in $z$ direction. The corresponding position-space variables for these cases are obviously the separation in time $t$ and distance $z$ of the quark and the antiquark in the operator in eq. (1.1). In the time-like case, the transition is performed with the help of the generic formula

$$
\begin{aligned}
\int \frac{d \omega}{2 \pi} e^{-i t \omega}(-2 \omega-i 0)^{-2 L \epsilon-n} & =\frac{e^{i(2 L \epsilon+n) \pi / 2}}{2 \Gamma(n+2 L \epsilon)} \theta(t)\left(\frac{t}{2}\right)^{2 L \epsilon+n-1} \\
& =\frac{i}{2 \Gamma(n+2 L \epsilon)} \theta(t)\left(\frac{i t}{2}\right)^{2 L \epsilon+n-1}
\end{aligned}
$$

where we assume $n$ to be integer.

Thus, renormalized time dependent correlation functions are expressed in terms of the following combination

$$
\ln \frac{i t e^{\gamma_{E}}}{2} \equiv \ln \frac{t}{2}+\gamma_{E}+i \frac{\pi}{2}
$$

where Euler's constant $\gamma_{E}$ appears naturally due to a universal factor,

$$
\Gamma(n+2 L \epsilon) \equiv \Gamma(1+2 L \epsilon)(1+2 L \epsilon)_{(n-1)},
$$

with $(1+2 L \epsilon)_{(n-1)}$ being the Pochhammer symbol. There is no $\gamma_{E}$ in momentum space results. The analogue of eq. (4.1) for the space-like case is

$$
\begin{aligned}
\int \frac{d p_{z}}{2 \pi} e^{i z p_{z}}\left(-2 p_{z}+i 0\right)^{-2 L \epsilon-n} & =\frac{e^{-i(2 L \epsilon+n) \pi / 2}}{2 \Gamma(n+2 L \epsilon)}\left(\frac{z}{2}\right)^{2 L \epsilon+n-1} \\
& =\frac{-i}{2 \Gamma(n+2 L \epsilon)}\left(\frac{-i z}{2}\right)^{2 L \epsilon+n-1} .
\end{aligned}
$$


Comparing eqs. (4.1) and (4.4), we infer that the transition from a time-like to a space-like renormalized correlation function in position space amounts to the formal substitution

$$
\ln \frac{i t}{2} \rightarrow \ln \frac{z}{2},
$$

up to a possible change of the global sign.

\section{Results}

In this section, we collect our results for the case of standard QCD with the $\mathrm{SU}(3)$ gauge group and $n_{f}$ active quarks triplets. Notice that the results for the self-energy and the propagator of the "heavy" field, the ADs $\gamma_{2}$ and $\gamma_{h}$ as well as the corresponding RCs are gauge dependent. The expressions below are given in Landau gauge, as it is most relevant for lattice applications. Full results for a generic gauge group and including the gauge as well as the momentum/position dependence are appended in the arxiv submission of this paper as auxiliary files in computer readable format. Many results given in the text and in the auxiliary files have originally been obtained by other authors and have been included here for completeness. In particular the $\mathrm{AD}$ of the heavy-light current $\gamma_{Q}$ was computed at one, two and three loops in refs. $[54,55]$, refs. $[12,56]$ and ref. [13], respectively. The AD $\gamma_{h}$ of the "heavy" field $h_{v}$ was computed at two and three loops in ref. [12] and refs. [13, 57], respectively, and, recently, at four loops in refs. [58, 59]. The RCs $Z_{3}, Z_{2}$ and $Z_{a}$ have been known through three loops for a long time [43-45]. The VEV $\Pi$ in eq. (1.1) was computed at two loops in ref. [60] and at three loops in ref. [61]. Notice that all these results for the ADs $\gamma_{h}, \gamma_{Q}$ and for the VEV $\Pi$ were obtained for the time-like choice of the vector $v^{\mu}$, with $v^{2}=1$. Our contribution is to clarify the changes for the space-time choice $v^{2}=-1$. We have also computed the VEV of the gluon off-light-cone operator in eq. (1.2) in the three-loop approximation as well the corresponding anomalous dimensions at two loops. Our results are in agreement with the two-loop VEV found in ref. [62] and the one-loop ADs first computed in refs. [37, 38].

\subsection{Anomalous dimensions and renormalization constants}

As follows from eq. (3.4), ADs and RCs are not sensitive to the sign choice of $v^{2}$. The analytic results for the $\mathrm{ADs} \beta, \gamma_{Q}, \gamma_{h}, \gamma_{2}, \gamma_{\perp \perp}$ and $\gamma_{\| \perp}$ are listed in appendix A, and those for the RCs $Z_{a}, Z_{Q}, Z_{h}, Z_{2}, Z_{\perp \perp}$ and $Z_{\| \perp}$ in appendix B.

\subsection{Correlation functions: momentum space}

Our result for the self-energy of the "heavy" field, $\Sigma_{h}$, defined in eq. (3.9) for the space-like case $v^{2}=-1$ can be written as ${ }^{5}$

$$
\begin{aligned}
\Sigma_{h}\left(\mu=2 \omega, v^{2}=-1\right) & =\Sigma_{h}^{t}+\left(\delta \Sigma_{h}^{s}\right)^{\operatorname{Re}}+\left(\delta \Sigma_{h}^{s}\right)^{\operatorname{Im}} \\
& =\sum_{n=1}^{3}\left(\Sigma_{h}^{t}\right)_{n} a^{n}+\sum_{n=1}^{3}\left(\delta \Sigma_{h}^{s}\right)_{n}^{\operatorname{Re}} a^{n}+i \sum_{n=1}^{3}\left(\delta \Sigma_{h}^{s}\right)_{n}^{\operatorname{Im}} a^{n},
\end{aligned}
$$

\footnotetext{
${ }^{5}$ In what follows, we do not show the trivial dependence of the self-energy $\Sigma_{h}(p)$ and the corresponding propagator $S_{h}$ on color indices. Furthermore, in eq. (5.13), we do not display the factor $\delta^{(D-1)}\left(x_{\perp}\right)$.
} 
where the first term corresponds to the time-like self-energy,

$$
\Sigma_{h}\left(\mu=-2 \omega, v^{2}=1\right)=\Sigma_{h}^{t}=\sum_{n=1}^{3}\left(\Sigma_{h}^{t}\right)_{n} a^{n}
$$

and the addenda $\delta \Sigma_{h}^{s}$ arise when going over to the space-like case using the substitution rule in eq. (3.43),

$$
\begin{aligned}
\ln ^{n}(-2 \omega) \rightarrow & \ln ^{n}(2 \omega)+\sum_{j=1}^{j \leq[n / 2]}\left(\frac{-\pi^{2}}{4}\right)^{j}\left(\begin{array}{c}
n \\
2 j
\end{array}\right) \ln ^{n-2 j}(2 \omega) \\
& -i \sum_{j=0}^{j \leq[(n-1) / 2]} \frac{\pi}{2}\left(\frac{-\pi^{2}}{4}\right)^{j}\left(\begin{array}{c}
n \\
2 j+1
\end{array}\right) \ln ^{n-2 j-1}(2 \omega) .
\end{aligned}
$$

Using Landau gauge, we find the following expressions:

$$
\begin{aligned}
\left(\Sigma_{h}{ }^{t}\right)_{1}= & -\frac{16}{3}, \quad\left(\Sigma_{h}{ }^{t}\right)_{2}=-\frac{4355}{18}-12 \pi^{2}+n_{f}\left[\frac{152}{9}+\frac{8}{9} \pi^{2}\right] \\
\left(\Sigma_{h}{ }^{t}\right)_{3}= & -\frac{3741889}{324}-\frac{8765}{9} \pi^{2}+\frac{4603}{360} \pi^{4}+893 \zeta_{3}+44 \pi^{2} \zeta_{3}-372 \zeta_{5} \\
& +n_{f}\left[\frac{388024}{243}+\frac{3316}{27} \pi^{2}-\frac{104}{135} \pi^{4}-120 \zeta_{3}\right]+n_{f}^{2}\left[-\frac{31232}{729}-\frac{256}{81} \pi^{2}+\frac{160}{27} \zeta_{3}\right] \\
\left(\delta \Sigma_{h}{ }^{s}\right)_{1}^{\mathrm{Re}}= & 0, \quad\left(\delta \Sigma_{h}{ }^{s}\right)_{2}^{\mathrm{Re}}=14 \pi^{2}-\frac{4}{3} \pi^{2} n_{f} \\
\left(\delta \Sigma_{h}{ }^{s}\right)_{3}^{\mathrm{Re}}= & \frac{2873}{3} \pi^{2}-\frac{1322}{9} \pi^{2} n_{f}+\frac{128}{27} \pi^{2} n_{f}^{2} \\
\left(\delta \Sigma_{h}{ }^{s}\right)_{1}^{\operatorname{Im}}= & -4 \pi, \quad\left(\delta \Sigma_{h}{ }^{s}\right)_{2}^{\operatorname{Im}}=-97 \pi+\frac{64}{9} \pi n_{f}, \\
\left(\delta \Sigma_{h}{ }^{s}\right)_{3}^{\operatorname{Im}}= & -\frac{188723}{36} \pi-132 \pi^{3}-\frac{8}{5} \pi^{5}-\frac{369}{2} \pi \zeta_{3}+n_{f}\left[\frac{20236}{27} \pi+\frac{160}{9} \pi^{3}+\frac{160}{3} \pi \zeta_{3}\right] \\
& +n_{f}^{2}\left[-\frac{1744}{81} \pi-\frac{16}{27} \pi^{3}\right] .
\end{aligned}
$$

Notice that the full dependence on $\ln [\mu /(2 \omega)]$ in eq. (5.4) can be easily restored from the evolution eq. (3.12) with the use of the $\mathrm{AD} \gamma_{h}$ as given in appendix $\mathrm{A}$.

Assuming $n_{f}=3$ and substituting $a_{s}=4 a \equiv \alpha_{s} / \pi$, we get numerically:

$$
\begin{aligned}
\left(\Sigma_{h}{ }^{t}\right)_{n_{f}=3} & =-1.33333 a_{s}-17.7121 a_{s}^{2}-180.297 a_{s}^{3}, \\
\left(\delta \Sigma_{h}{ }^{s}\right)_{n_{f}=3}^{\mathrm{Re}} & =6.1685 a_{s}^{2}+86.3076 a_{s}^{3}, \\
\left(\delta \Sigma_{h}{ }^{s}\right)_{n_{f}=3}^{\operatorname{Im}} & =-3.14159 a_{s}-14.8571 a_{s}^{2}-206.265 a_{s}^{3} .
\end{aligned}
$$

Notice that the heavy-field self-energy at $v^{2}=-1$ acquires a large imaginary part, whereas the difference in the real part is minor. 
Next, we consider the momentum-space correlation function in eq. (3.18). Our result for $\tilde{D}$ reads $^{6}$

$$
\begin{aligned}
\pi^{2} \tilde{D}\left(\mu=2 \omega, v^{2}=-1\right) & =-\tilde{D}^{t}+\left(\delta \tilde{D}^{s}\right)^{\mathrm{Re}}+\left(\delta \tilde{D}^{s}\right)^{\operatorname{Im}} \\
& =-\sum_{n=0}^{2}\left(\tilde{D}^{t}\right)_{n} a^{n}+\sum_{n=0}^{2}\left(\delta \tilde{D}^{s}\right)_{n}^{\operatorname{Re}} a^{n}+i \sum_{n=0}^{2}\left(\delta \tilde{D}^{s}\right)_{n}^{\operatorname{Im}} a^{n}
\end{aligned}
$$

where, as above, the first term corresponds to the time-like correlation function,

$$
\pi^{2} \tilde{D}\left(\mu=-2 \omega, v^{2}=1\right)=\tilde{D}^{t}=\sum_{n=0}^{2}\left(\tilde{D}^{t}\right)_{n} a^{n} .
$$

The coefficients in eq. (5.6) are given by

$$
\begin{aligned}
\left(\tilde{D}^{t}\right)_{1}=68+\frac{16}{3} \pi^{2} & =3, \\
\left(\tilde{D}^{t}\right)_{2} & =\frac{24749}{6}+\frac{3680}{9} \pi^{2}-\frac{32}{27} \pi^{4}-\frac{3872}{3} \zeta_{3}+n_{f}\left[-\frac{1849}{9}-\frac{328}{27} \pi^{2}+64 \zeta_{3}\right] \\
\left(\delta \tilde{D}^{s}\right)_{0}^{\mathrm{Re}} & =0, \quad\left(\delta \tilde{D}^{s}\right)_{1}^{\mathrm{Re}}=0 \\
\left(\delta \tilde{D}^{s}\right)_{2}^{\mathrm{Re}} & =90 \pi^{2}-4 \pi^{2} n_{f}, \quad\left(\delta \tilde{D}^{s}\right)_{1}^{\operatorname{Im}}=-12 \pi \\
\left(\delta \tilde{D}^{s}\right)_{0}^{\mathrm{Im}} & =0, \\
\left(\delta \tilde{D}^{s}\right)_{2}^{\operatorname{Im}} & =-\frac{3314}{3} \pi-\frac{776}{9} \pi^{3}+n_{f}\left[52 \pi+\frac{32}{9} \pi^{3}\right] .
\end{aligned}
$$

For $n_{f}=3$, we have numerically

$$
\begin{aligned}
& \left(\delta \tilde{D}^{t}\right)_{n_{f}=3}=3 .+30.1595 a_{s}+359.267 a_{s}^{2} \\
& \left(\delta \tilde{D}^{s}\right)_{n_{f}=3}^{\mathrm{Re}}=48.1143 a_{s}^{2} \\
& \left(\delta \tilde{D}^{s}\right)_{n_{f}=3}^{\mathrm{Im}}=-9.42478 a_{s}-332.689 a_{s}^{2} .
\end{aligned}
$$

Finally, we present below our results for the two momentum-space correlators,

$$
\begin{aligned}
\tilde{D}_{\perp \perp}\left(\mu=2 \omega, v^{2}=-1\right) & =a\left(\tilde{D}_{\perp \perp}^{t}+\left(\delta \tilde{D}_{\perp \perp}^{s}\right)^{\mathrm{Re}}+\left(\delta \tilde{D}_{\perp \perp}^{s}\right)^{\mathrm{Im}}\right) \\
& =a\left(\sum_{n=0}^{2}\left(\tilde{D}_{\perp \perp}^{t}\right)_{n} a^{n}+\sum_{n=0}^{3}\left(\delta \tilde{D}_{\perp \perp}^{s}\right)_{n}^{\mathrm{Re}} a^{n}+i \sum_{n=0}^{2}\left(\delta \tilde{D}_{\perp \perp}^{s}\right)_{n}^{\operatorname{Im}} a^{n}\right), \\
\tilde{D}_{\| \perp}\left(\mu=2 \omega, v^{2}=-1\right) & =a\left(\tilde{D}_{\| \perp}^{t}+\left(\delta \tilde{D}_{\| \perp}^{s}\right)^{\mathrm{Re}}+\left(\delta \tilde{D}_{\| \perp}^{s}\right)^{\mathrm{Im}}\right) \\
& =a\left(\sum_{n=0}^{2}\left(\tilde{D}_{\| \perp}^{t}\right)_{n} a^{n}+\sum_{n=0}^{3}\left(\delta \tilde{D}_{\| \perp}^{s}\right)_{n}^{\mathrm{Re}} a^{n}+i \sum_{n=0}^{2}\left(\delta \tilde{D}_{\| \perp}^{s}\right)_{n}^{\operatorname{Im}} a^{n}\right),
\end{aligned}
$$

\footnotetext{
${ }^{6}$ Notice that there is an overall minus sign between the time-like and space-like correlation functions, unlike for the self-energy $\Sigma_{h}$.
} 
where

$$
\begin{aligned}
& \left(\tilde{D}_{\perp \perp}^{t}\right)_{0}=\frac{64}{3}, \\
& \left(\tilde{D}_{\perp \perp}^{t}\right)_{1}=\frac{8576}{9}+\frac{256}{3} \pi^{2}-\frac{1664}{27} n_{f}, \\
& \left(\tilde{D}_{\perp \perp}^{t}\right)_{2}=\frac{1994336}{27}+\frac{94208}{9} \pi^{2}-19584 \zeta_{3}+n_{f}\left[-\frac{692224}{81}-\frac{15616}{27} \pi^{2}+\frac{8960}{9} \zeta_{3}\right] \\
& +n_{f}^{2}\left[\frac{53504}{243}+\frac{512}{81} \pi^{2}\right], \\
& \left(\delta \tilde{D}_{\perp \perp}^{s}\right)_{0}^{\mathrm{Re}}=0 \\
& \left(\delta \tilde{D}_{\perp \perp}^{s}\right)_{1}^{\mathrm{Re}}=0, \\
& \left(\delta \tilde{D}_{\perp \perp}^{s}\right)_{2}^{\mathrm{Re}}=-\frac{2560}{3} \pi^{2}+\frac{1664}{9} \pi^{2} n_{f}-\frac{256}{27} \pi^{2} n_{f}^{2}, \\
& \left(\delta \tilde{D}_{\perp \perp}^{s}\right)_{0}^{\operatorname{Im}}=0 \text {, } \\
& \left(\delta \tilde{D}_{\perp \perp}^{s}\right)_{1}^{\operatorname{Im}}=\frac{320}{3} \pi-\frac{128}{9} \pi n_{f}, \\
& \left(\delta \tilde{D}_{\perp \perp}^{s}\right)_{2}^{\operatorname{Im}}=\frac{143744}{9} \pi+\frac{4864}{3} \pi^{3}+n_{f}\left[-\frac{63232}{27} \pi-\frac{1024}{9} \pi^{3}\right]+\frac{6656}{81} \pi n_{f}^{2}, \\
& \left(\tilde{D}_{\| \perp}^{t}\right)_{0}=-\frac{64}{3}, \\
& \left(\tilde{D}_{\| \perp}^{t}\right)_{1}=-\frac{9536}{9}-\frac{256}{3} \pi^{2}+\frac{1280}{27} n_{f}, \\
& \left(\tilde{D}_{\| \perp}^{t}\right)_{2}=-\frac{2253920}{27}-\frac{97088}{9} \pi^{2}+21888 \zeta_{3}+n_{f}\left[\frac{638656}{81}+\frac{14848}{27} \pi^{2}-\frac{8960}{9} \zeta_{3}\right] \\
& +n_{f}^{2}\left[-\frac{33536}{243}-\frac{512}{81} \pi^{2}\right] \\
& \left(\delta \tilde{D}_{\| \perp}^{s}\right)_{0}^{\mathrm{Re}}=0 \\
& \left(\delta \tilde{D}_{\| \perp}^{s}\right)_{1}^{\operatorname{Re}}=0 \\
& \left(\delta \tilde{D}_{\| \perp}^{s}\right)_{2}^{\mathrm{Re}}=\frac{7744}{3} \pi^{2}-\frac{2816}{9} \pi^{2} n_{f}+\frac{256}{27} \pi^{2} n_{f}^{2}, \\
& \left(\delta \tilde{D}_{\| \perp}^{s}\right)_{0}^{\operatorname{Im}}=0 \\
& \left(\delta \tilde{D}_{\| \perp}^{s}\right)_{1}^{\operatorname{Im}}=-\frac{704}{3} \pi+\frac{128}{9} \pi n_{f}, \\
& \left(\delta \tilde{D}_{\| \perp}^{s}\right)_{2}^{\operatorname{Im}}=-\frac{229376}{9} \pi-\frac{6400}{3} \pi^{3}+n_{f}\left[\frac{73600}{27} \pi+\frac{1024}{9} \pi^{3}\right]-\frac{5120}{81} \pi n_{f}^{2} .
\end{aligned}
$$

For $n_{f}=3$, one obtains numerically:

$$
\begin{aligned}
& \left(\delta \tilde{D}_{\perp \perp}^{t}\right)_{n_{f}=3}=\frac{64}{3}\left(1+18.8696 a_{s}+342.786 a_{s}^{2}\right), \\
& \left(\delta \tilde{D}_{\perp \perp}^{s}\right)_{n_{f}=3}^{\mathrm{Re}}=-236.871 a_{s}^{2} \\
& \left(\delta \tilde{D}_{\perp \perp}^{s}\right)_{n_{f}=3}^{\mathrm{Im}}=50.2655 a_{s}+4382.21 a_{s}^{2} \\
& \left(\delta \tilde{D}_{\| \perp}^{t}\right)_{n_{f}=3}=-\frac{64}{3}\left(1+20.6196 a_{s}+378.205 a_{s}^{2}\right),
\end{aligned}
$$




$$
\begin{aligned}
& \left(\delta \tilde{D}_{\| \perp}^{s}\right)_{n_{f}=3}^{\operatorname{Re}}=1065.92 a_{s}^{2}, \\
& \left(\delta \tilde{D}_{\| \perp}^{s}\right)_{n_{f}=3}^{\operatorname{Im}}=-150.796 a_{s}-6982.91 a_{s}^{2} .
\end{aligned}
$$

\subsection{Correlation functions: position space}

As follows from eq. (4.5), the time-like and space-like renormalized correlation functions in position space are given by identical expressions with the substitution $i t \rightarrow z$. Our result for the "heavy" propagator in position space is in agreement with eq. (12) of ref. [13] derived for $v^{2}=1$. For the correlation function in eq. (1.1), we obtain

$$
\Pi\left(z>0, v^{2}=-1\right)=\frac{6}{i \pi^{2} z^{3}}\left(1+\sum_{n=1}^{3}(F)_{n} a^{n}\right),
$$

with coefficients

$$
\begin{aligned}
(F)_{1}= & \frac{32}{3}+\frac{16}{9} \pi^{2}+8 L_{z} \\
(F)_{2}= & \frac{7025}{18}+\frac{812}{27} \pi^{2}-\frac{32}{81} \pi^{4}-\frac{3872}{9} \zeta_{3}+\frac{3388}{9} L_{z}+\frac{1552}{27} \pi^{2} L_{z}+120 L_{z}^{2} \\
& +n_{f}\left[-\frac{589}{27}+\frac{32}{81} \pi^{2}+\frac{64}{3} \zeta_{3}-\frac{56}{3} L_{z}-\frac{64}{27} \pi^{2} L_{z}-\frac{16}{3} L_{z}^{2}\right]
\end{aligned}
$$

where $L_{z}=\ln \left(\mu \mathrm{e}^{\gamma_{E}} z / 2\right)$. For $n_{f}=3$, we get numerically:

$$
(F)_{n_{f}=3}=1.0+7.05316 a_{s}+2.0 L_{z} a_{s}+9.66546 a_{s}^{2}+51.0988 L_{z} a_{s}^{2}+6.5 L_{z}^{2} a_{s}^{2} .
$$

Notice that the higher-order coefficients in position space are considerably smaller than in momentum space, cf. eq. (5.5).

Our results for the position-space functions $\Pi_{\perp \perp}$ and $\Pi_{\| \perp}$,

$$
\begin{aligned}
& \Pi_{\perp \perp}\left(z>0, v^{2}=-1\right)=\frac{128 a}{z^{4}}\left(1+\sum_{n=1}^{2}\left(F_{\perp \perp}\right)_{n} a^{n}\right), \\
& \Pi_{\| \perp}\left(z>0, v^{2}=-1\right)=-\frac{128 a}{z^{4}}\left(1+\sum_{n=1}^{2}\left(F_{\| \perp}\right)_{n} a^{n}\right),
\end{aligned}
$$

are given by

$$
\begin{aligned}
\left(F_{\perp \perp}\right)_{1}= & \frac{79}{3}+4 \pi^{2}+10 L_{z}+n_{f}\left[-\frac{4}{9}-\frac{4}{3} L_{z}\right] \\
\left(F_{\perp \perp}\right)_{2}= & \frac{18671}{18}+\frac{556}{3} \pi^{2}-918 \zeta_{3}+\frac{2732}{3} L_{z}+152 \pi^{2} L_{z}+160 L_{z}^{2} \\
& +n_{f}\left[-\frac{1820}{27}-\frac{16}{9} \pi^{2}+\frac{140}{3} \zeta_{3}-\frac{832}{9} L_{z}-\frac{32}{3} \pi^{2} L_{z}-\frac{104}{3} L_{z}^{2}\right] \\
& +n_{f}^{2}\left[-\frac{20}{81}+\frac{32}{27} L_{z}+\frac{16}{9} L_{z}^{2}\right] \\
\left(F_{\| \perp}\right)_{1}= & \frac{28}{3}+4 \pi^{2}+22 L_{z}+n_{f}\left[\frac{2}{9}-\frac{4}{3} L_{z}\right]
\end{aligned}
$$




$$
\begin{aligned}
\left(F_{\| \perp}\right)_{2}= & \frac{9011}{18}+\frac{175}{3} \pi^{2}-1026 \zeta_{3}+\frac{1844}{3} L_{z}+200 \pi^{2} L_{z}+484 L_{z}^{2} \\
& +n_{f}\left[-\frac{497}{27}+\frac{32}{9} \pi^{2}+\frac{140}{3} \zeta_{3}-\frac{364}{9} L_{z}-\frac{32}{3} \pi^{2} L_{z}-\frac{176}{3} L_{z}^{2}\right] \\
& +n_{f}^{2}\left[-\frac{68}{81}-\frac{16}{27} L_{z}+\frac{16}{9} L_{z}^{2}\right] .
\end{aligned}
$$

For $n_{f}=3$, one obtains numerically:

$$
\begin{aligned}
& \left(F_{\perp \perp}\right)_{n_{f}=3}=1+16.1196 a_{s}+1.5 L_{z} a_{s}+104.635 a_{s}^{2}+114.272 L_{z} a_{s}^{2}+4.5 L_{z}^{2} a_{s}^{2} \\
& \left(F_{\| \perp}\right)_{n_{f}=3}=1+12.3696 a_{s}+4.5 L_{z} a_{s}+3.36335 a_{s}^{2}+134.131 L_{z} a_{s}^{2}+20.25 L_{z}^{2} a_{s}^{2} .
\end{aligned}
$$

In the case of QED, the gluon non-local operator in eq. (1.2) contracted with $g^{\mu \alpha} g^{\nu \beta}$ can be interpreted as a "photonic condensate" regulated with the splitting technique [63]. In terms of the scalar functions $\Pi_{\perp \perp}$ and $\Pi_{\| \perp}$, we have

$$
\mathbb{G}_{\mu \nu}^{\mathrm{QED}, \mu \nu}(z)=6\left(\Pi_{\perp \perp}+\Pi_{\| \perp}\right)=-\frac{1}{8 z^{4}}\left(\frac{\alpha_{e}}{\pi}\right)^{2}\left[1+\left(\frac{3}{4}-\frac{4}{9} n_{f}-\frac{4}{3} n_{f} L_{z}\right) \frac{\alpha_{e}}{\pi}\right],
$$

where $\alpha_{e}$ is the fine-structure constant. Notice that, in the QED case, the Wilson line appearing in eq. (1.2) then reduces to just 1. Thus, $\mathbb{G}_{\mu \nu}^{\mathrm{QED}, \mu \nu}(z)$ is directly expressible in terms of the photon propagator in position space. The latter is currently known through order $\alpha_{e}^{5}[64,65]$.

\section{Conclusions}

We have studied the renormalization and vacuum expectation values of non-local off-lightcone operators of a quark and an antiquark field, eq. (1.1), and also of two gluon field strength tensors, eq. (1.2), connected by a straight-line-ordered Wilson line, eq. (1.3).

Nucleon matrix elements of these operators are usually called qPDFs and they are amenable to nonperturbative calculations on the lattice for space-like separations of the quark fields. At the same time, they are counterparts of similar time-like matrix elements that have been discussed in the past in the context of heavy-quark expansion in $B$-meson weak decays, and it is important to understand the relation between time-like and space-like renormalization and matrix elements.

We have shown, to all orders in perturbation theory, that the results for a generic Green function involving a qPDF operator at space-like and time-like separations are related by a specific substitution rule reflecting analytic continuation in the square $v^{2}$ of the fourvector $v^{\mu}$ pointing along the Wilson line; see eq. (3.43). The RCs and ADs are the same for space-like and time-like separations. This result is also relevant in the context of TMD factorization, where Wilson lines are shifted off the light cone to regularize rapidity divergences in TMD operators [16]. Our statement is that the ADs and RCs do not depend on the direction of the shift, space-like or time-like.

We have calculated the self-energy of the "heavy" field $h_{v}$ in the effective field theory of eq. (2.1), the quark-antiquark qPDF $\mathrm{AD}$ and $\mathrm{VEV}$, and all the RCs and ADs that are 
involved in the respective renormalization through three loops in the $\overline{\mathrm{MS}}$ scheme. Our results agree with the literature as far as it goes. In addition, we have clarified the general RG pattern for the gluon qPDF operator in eq. (1.2) and calculated its VEV through three loops, from which the two-loop ADs can be extracted avoiding pollution by gaugenoninvariant operators.

Our results can be used in lattice calculations aiming at the determination of quark and gluon PDFs, e.g., in the nucleon, if the linear UV divergences of lattice observables are removed by considering suitable ratios of matrix elements involving the same operator $[34,35]$.

\section{Acknowledgments}

We thank Andrey Grozin for reading the manuscript and valuable advice. The work of V.M.B. and B.A.K. was supported in part by the DFG Research Unit FOR 2926 under Grant No. 409651613. The work of K.G.C. was supported in part by DFG grant CH 1479/2-1.

\section{A Anomalous dimensions}

Representing a generic $\operatorname{AD} \gamma(a)$ as

$$
\gamma=\sum_{n \geq 1}(\gamma)_{n} a^{n}
$$

we have for the coefficients relevant here

$$
\begin{aligned}
(\beta)_{1} & =-11+\frac{2}{3} n_{f} \\
(\beta)_{2} & =-102+\frac{38}{3} n_{f} \\
(\beta)_{3} & =-\frac{2857}{2}+\frac{5033}{18} n_{f}-\frac{325}{54} n_{f}^{2} \\
\left(\gamma_{Q}\right)_{1} & =2 \\
\left(\gamma_{Q}\right)_{2} & =\frac{127}{9}+\frac{28}{27} \pi^{2}-\frac{10}{9} n_{f}, \\
\left(\gamma_{Q}\right)_{3} & =-\frac{61}{6}+\frac{1372}{81} \pi^{2}+\frac{760}{243} \pi^{4}-\frac{356}{9} \zeta_{3}+n_{f}\left[-\frac{344}{81}-\frac{392}{243} \pi^{2}-\frac{664}{27} \zeta_{3}\right]-\frac{70}{81} n_{f}^{2}, \\
\left(\gamma_{h}\right)_{1} & =4 \\
\left(\gamma_{h}\right)_{2} & =\frac{179}{3}-\frac{32}{9} n_{f}, \\
\left(\gamma_{h}\right)_{3} & =\frac{23815}{36}+\frac{8}{5} \pi^{4}+\frac{369}{2} \zeta_{3}+n_{f}\left[-\frac{2006}{27}-\frac{160}{3} \zeta_{3}\right]-\frac{80}{81} n_{f}^{2}, \\
\left(\gamma_{2}\right)_{1} & =0 \\
\left(\gamma_{2}\right)_{2} & =-\frac{67}{3}+\frac{4}{3} n_{f},
\end{aligned}
$$




$$
\begin{aligned}
\left(\gamma_{2}\right)_{3} & =-\frac{20729}{36}+\frac{79}{2} \zeta_{3}+\frac{550}{9} n_{f}-\frac{20}{27} n_{f}^{2} \\
\left(\gamma_{\perp \perp}\right)_{1} & =-3 \\
\left(\gamma_{\perp \perp}\right)_{2} & =-34+6 \pi^{2}+\frac{13}{3} n_{f}, \\
\left(\gamma_{\| \perp}\right)_{1} & =0 \\
\left(\gamma_{\| \perp}\right)_{2} & =6 \pi^{2} .
\end{aligned}
$$

\section{B Renormalization constants}

Representing a generic $\mathrm{RC} Z(a, \epsilon)$ as

$$
Z(a, \epsilon)=1+\sum_{n \geq 1}(Z(\epsilon))_{n}\left(\frac{a}{\epsilon}\right)^{n}
$$

we have for the coefficients relevant here

$$
\begin{aligned}
\left(Z_{a}\right)_{1}= & -11+\frac{2}{3} n_{f}, \\
\left(Z_{a}\right)_{2}= & 121-51 \epsilon+n_{f}\left[-\frac{44}{3}+\frac{19}{3} \epsilon\right]+\frac{4}{9} n_{f}^{2} \\
\left(Z_{a}\right)_{3}= & -1331+1309 \epsilon-\frac{2857}{6} \epsilon^{2}+n_{f}\left[242-\frac{2177}{9} \epsilon+\frac{5033}{54} \epsilon^{2}\right] \\
& +n_{f}^{2}\left[-\frac{44}{3}+\frac{266}{27} \epsilon-\frac{325}{162} \epsilon^{2}\right]+\frac{8}{27} n_{f}^{3}, \\
\left(Z_{Q}\right)_{1}= & -2, \\
\left(Z_{Q}\right)_{2}= & 13-\frac{127}{18} \epsilon-\frac{14}{27} \pi^{2} \epsilon+n_{f}\left[-\frac{2}{3}+\frac{5}{9} \epsilon\right] \\
\left(Z_{Q}\right)_{3}= & -104+\frac{3614}{27} \epsilon+\frac{392}{81} \pi^{2} \epsilon+\frac{61}{18} \epsilon^{2}-\frac{1372}{243} \pi^{2} \epsilon^{2}-\frac{760}{729} \pi^{4} \epsilon^{2}+\frac{356}{27} \zeta_{3} \epsilon^{2} \\
& +n_{f}\left[\frac{100}{9}-\frac{1358}{81} \epsilon-\frac{56}{243} \pi^{2} \epsilon+\frac{344}{243} \epsilon^{2}+\frac{392}{729} \pi^{2} \epsilon^{2}+\frac{664}{81} \zeta_{3} \epsilon^{2}\right] \\
& +n_{f}^{2}\left[-\frac{8}{27}+\frac{20}{81} \epsilon+\frac{70}{243} \epsilon^{2}\right], \\
\left(Z_{h}\right)_{1}= & 4, \\
\left(Z_{h}\right)_{2}= & -14+\frac{179}{6} \epsilon+n_{f}\left[\frac{4}{3}-\frac{16}{9} \epsilon\right], \\
\left(Z_{h}\right)_{3}= & 84-\frac{2119}{9} \epsilon+\frac{23815}{108} \epsilon^{2}+\frac{8}{15} \pi^{4} \epsilon^{2}+\frac{123}{2} \zeta_{3} \epsilon^{2} \\
& +n_{f}\left[-\frac{128}{9}+\frac{974}{27} \epsilon-\frac{2006}{81} \epsilon^{2}-\frac{160}{9} \zeta_{3} \epsilon^{2}\right]+n_{f}^{2}\left[\frac{16}{27}-\frac{64}{81} \epsilon-\frac{80}{243} \epsilon^{2}\right] \\
\left(Z_{2}\right)_{3}= & \frac{737}{9} \epsilon-\frac{20729}{108} \epsilon^{2}+\frac{79}{6} \zeta_{3} \epsilon^{2}+n_{f}\left[-\frac{266}{27} \epsilon+\frac{550}{27} \epsilon^{2}\right]+n_{f}^{2}\left[\frac{8}{27} \epsilon-\frac{20}{81} \epsilon^{2}\right], \\
\left(Z_{2}\right)_{2}= & -\frac{67}{6} \epsilon+\frac{2}{3} \epsilon n_{f}, \\
& 0
\end{aligned}
$$




$$
\begin{aligned}
& \left(Z_{\perp \perp}\right)_{1}=3, \\
& \left(Z_{\perp \perp}\right)_{2}=-12+\left(17-3 \pi^{2}\right) \epsilon+n_{f}\left[1-\frac{13}{6} \epsilon\right], \\
& \left(Z_{\| \perp}\right)_{1}=0, \\
& \left(Z_{\| \perp}\right)_{2}=-3 \pi^{2} \epsilon .
\end{aligned}
$$

Open Access. This article is distributed under the terms of the Creative Commons Attribution License (CC-BY 4.0), which permits any use, distribution and reproduction in any medium, provided the original author(s) and source are credited.

\section{References}

[1] Y. Makeenko and A.A. Migdal, Quantum Chromodynamics as Dynamics of Loops, Sov. J. Nucl. Phys. 32 (1980) 431 [inSPIRE].

[2] A.M. Polyakov, Gauge Fields as Rings of Glue, Nucl. Phys. B 164 (1980) 171 [InSPIRE].

[3] J.-L. Gervais and A. Neveu, The Slope of the Leading Regge Trajectory in Quantum Chromodynamics, Nucl. Phys. B 163 (1980) 189 [INSPIRE].

[4] V.S. Dotsenko and S.N. Vergeles, Renormalizability of Phase Factors in the Nonabelian Gauge Theory, Nucl. Phys. B 169 (1980) 527 [INSPIRE].

[5] N.S. Craigie and H. Dorn, On the Renormalization and Short Distance Properties of Hadronic Operators in QCD, Nucl. Phys. B 185 (1981) 204 [INSPIRE].

[6] I. Arefeva, Quantum contour field equations, Phys. Lett. B 93 (1980) 347 [INSPIRE].

[7] R.A. Brandt, F. Neri and M.-a. Sato, Renormalization of Loop Functions for All Loops, Phys. Rev. D 24 (1981) 879 [inSPIRE].

[8] H. Dorn, Renormalization of Path Ordered Phase Factors and Related Hadron Operators in Gauge Field Theories, Fortsch. Phys. 34 (1986) 11 [InSPIRE].

[9] G.P. Korchemsky and A.V. Radyushkin, Loop Space Formalism and Renormalization Group for the Infrared Asymptotics of QCD, Phys. Lett. B 171 (1986) 459 [InSPIRE].

[10] G.P. Korchemsky and A.V. Radyushkin, Renormalization of the Wilson Loops Beyond the Leading Order, Nucl. Phys. B 283 (1987) 342 [inSPIRE].

[11] G.P. Korchemsky and A.V. Radyushkin, Infrared factorization, Wilson lines and the heavy quark limit, Phys. Lett. B 279 (1992) 359 [hep-ph/9203222] [INSPIRE].

[12] D.J. Broadhurst and A.G. Grozin, Two loop renormalization of the effective field theory of a static quark, Phys. Lett. B 267 (1991) 105 [hep-ph/9908362] [INSPIRE].

[13] K.G. Chetyrkin and A.G. Grozin, Three loop anomalous dimension of the heavy light quark current in HQET, Nucl. Phys. B 666 (2003) 289 [hep-ph/0303113] [INSPIRE].

[14] A.G. Grozin, T. Huber and D. Maître, On one master integral for three-loop on-shell HQET propagator diagrams with mass, JHEP 07 (2007) 033 [arXiv: 0705.2609] [INSPIRE].

[15] A.G. Grozin and R.N. Lee, Three-loop HQET vertex diagrams for $B^{0}-\bar{B}^{0}$ mixing, JHEP 02 (2009) 047 [arXiv: 0812.4522] [INSPIRE].

[16] J. Collins, Foundations of perturbative QCD, Camb. Monogr. Part. Phys. Nucl. Phys. Cosmol. 32 (2011) 1-624 [INSPIRE]. 
[17] X. Ji, Parton Physics on a Euclidean Lattice, Phys. Rev. Lett. 110 (2013) 262002 [arXiv: 1305.1539] [INSPIRE].

[18] A.V. Radyushkin, Quasi-parton distribution functions, momentum distributions and pseudo-parton distribution functions, Phys. Rev. D 96 (2017) 034025 [arXiv:1705.01488] [INSPIRE].

[19] T. Izubuchi, X. Ji, L. Jin, I.W. Stewart and Y. Zhao, Factorization Theorem Relating Euclidean and Light-Cone Parton Distributions, Phys. Rev. D 98 (2018) 056004 [arXiv: 1801.03917] [INSPIRE].

[20] H.-W. Lin et al., Parton distributions and lattice QCD calculations: a community white paper, Prog. Part. Nucl. Phys. 100 (2018) 107 [arXiv:1711.07916] [InSPIRE].

[21] K. Cichy and M. Constantinou, A guide to light-cone PDFs from Lattice QCD: an overview of approaches, techniques and results, Adv. High Energy Phys. 2019 (2019) 3036904 [arXiv: 1811.07248] [INSPIRE].

[22] W. Detmold and C.J. Lin, Deep-inelastic scattering and the operator product expansion in lattice QCD, Phys. Rev. D 73 (2006) 014501 [hep-lat/0507007] [InSPIRE].

[23] V. Braun and D. Müller, Exclusive processes in position space and the pion distribution amplitude, Eur. Phys. J. C 55 (2008) 349 [arXiv:0709.1348] [INSPIRE].

[24] Y.-Q. Ma and J.-W. Qiu, Exploring Partonic Structure of Hadrons Using ab initio Lattice QCD Calculations, Phys. Rev. Lett. 120 (2018) 022003 [arXiv: 1709. 03018] [INSPIRE].

[25] X. Xiong, X. Ji, J.-H. Zhang and Y. Zhao, One-loop matching for parton distributions: Nonsinglet case, Phys. Rev. D 90 (2014) 014051 [arXiv:1310.7471] [INSPIRE].

[26] X. Ji and J.-H. Zhang, Renormalization of quasiparton distribution, Phys. Rev. D 92 (2015) 034006 [arXiv: 1505.07699] [INSPIRE].

[27] X. Ji, J.-H. Zhang and Y. Zhao, Renormalization in Large Momentum Effective Theory of Parton Physics, Phys. Rev. Lett. 120 (2018) 112001 [arXiv:1706.08962] [InSPIRE].

[28] T. Ishikawa, Y.-Q. Ma, J.-W. Qiu and S. Yoshida, Renormalizability of quasiparton distribution functions, Phys. Rev. D 96 (2017) 094019 [arXiv: 1707.03107] [InSPIRE].

[29] W. Wang and S. Zhao, On the power divergence in quasi gluon distribution function, JHEP 05 (2018) 142 [arXiv: 1712.09247] [INSPIRE].

[30] W. Wang, S. Zhao and R. Zhu, Gluon quasidistribution function at one loop, Eur. Phys. J. C 78 (2018) 147 [arXiv: 1708. 02458] [INSPIRE].

[31] J.-H. Zhang, X. Ji, A. Schäfer, W. Wang and S. Zhao, Accessing Gluon Parton Distributions in Large Momentum Effective Theory, Phys. Rev. Lett. 122 (2019) 142001 [arXiv: 1808.10824] [INSPIRE].

[32] W. Wang, J.-H. Zhang, S. Zhao and R. Zhu, Complete matching for quasidistribution functions in large momentum effective theory, Phys. Rev. D 100 (2019) 074509 [arXiv: 1904.00978] [INSPIRE].

[33] I. Balitsky, W. Morris and A. Radyushkin, Gluon Pseudo-Distributions at Short Distances: Forward Case, arXiv:1910.13963 [INSPIRE].

[34] K. Orginos, A. Radyushkin, J. Karpie and S. Zafeiropoulos, Lattice QCD exploration of parton pseudo-distribution functions, Phys. Rev. D 96 (2017) 094503 [arXiv:1706.05373] [INSPIRE]. 
[35] V.M. Braun, A. Vladimirov and J.-H. Zhang, Power corrections and renormalons in parton quasidistributions, Phys. Rev. D 99 (2019) 014013 [arXiv:1810.00048] [InSPIRE].

[36] A. Di Giacomo, H.G. Dosch, V.I. Shevchenko and Y. Simonov, Field correlators in QCD: Theory and applications, Phys. Rept. 372 (2002) 319 [hep-ph/0007223] [INSPIRE].

[37] H. Dorn and E. Wieczorek, Renormalization and Short Distance Properties of String Type Equations in QCD, Z. Phys. C 9 (1981) 49 [Erratum ibid. 9 (1981) 274] [InSPIRE].

[38] H. Dorn, D. Robaschik and E. Wieczorek, Renormalization and short distance properties of gauge invariant gluoinum and hadron operators, Annalen Phys. 40 (1983) 166.

[39] M. Neubert, Heavy quark symmetry, Phys. Rept. 245 (1994) 259 [hep-ph/9306320] [INSPIRE].

[40] G.M. Cicuta and E. Montaldi, Analytic renormalization via continuous space dimension, Lett. Nuovo Cim. 4 (1972) 329 [inSPIRE].

[41] J.F. Ashmore, A Method of Gauge Invariant Regularization, Lett. Nuovo Cim. 4 (1972) 289 [INSPIRE].

[42] G. 't Hooft and M.J.G. Veltman, Regularization and Renormalization of Gauge Fields, Nucl. Phys. B 44 (1972) 189 [INSPIRE].

[43] O.V. Tarasov, A.A. Vladimirov and A. Zharkov, The Gell-Mann-Low Function of QCD in the Three Loop Approximation, Phys. Lett. B 93 (1980) 429 [INSPIRE].

[44] O.V. Tarasov, Anomalous dimensions of quark masses in the three-loop approximation, Phys. Part. Nucl. Lett. 17 (2020) 109 [arXiv:1910.12231] [INSPIRE].

[45] S.A. Larin and J.A.M. Vermaseren, The Three loop QCD $\beta$-function and anomalous dimensions, Phys. Lett. B 303 (1993) 334 [hep-ph/9302208] [INSPIRE].

[46] A.G. Grozin, Heavy quark effective theory, Springer Tracts Mod. Phys. 201 (2004) 1.

[47] P. Nogueira, Automatic Feynman graph generation, J. Comput. Phys. 105 (1993) 279.

[48] A.V. Smirnov and F.S. Chuharev, FIRE6: Feynman Integral REduction with Modular Arithmetic, Comput. Phys. Commun. 247 (2020) 106877 [arXiv:1901.07808] [INSPIRE].

[49] R.N. Lee, Presenting LiteRed: a tool for the Loop InTEgrals REDuction, arXiv:1212.2685 [INSPIRE].

[50] R.N. Lee, LiteRed 1.4: a powerful tool for reduction of multiloop integrals, J. Phys. Conf. Ser. 523 (2014) 012059 [arXiv: 1310.1145] [inSPIRE].

[51] A.G. Grozin, Calculating three loop diagrams in heavy quark effective theory with integration by parts recurrence relations, JHEP 03 (2000) 013 [hep-ph/0002266] [INSPIRE].

[52] A.G. Grozin, Higher radiative corrections in HQET, in Helmholz International Summer School on Heavy Quark Physics, pp. 55-88, 9, 2008 [arXiv:0809.4540] [INSPIRE].

[53] V.A. Smirnov, Analytic tools for Feynman integrals, Springer Tracts Mod. Phys. 250 (2012) 1.

[54] H. Politzer and M.B. Wise, Leading Logarithms of Heavy Quark Masses in Processes with Light and Heavy Quarks, Phys. Lett. B 206 (1988) 681 [INSPIRE].

[55] M.A. Shifman and M.B. Voloshin, On the annihilation constants of mesons consisting of a heavy and a light quark, and $B^{0} \leftrightarrow \bar{B}^{0}$ oscillations, Sov. J. Nucl. Phys. 45 (1987) 292 [INSPIRE]. 
[56] X.-D. Ji and M.J. Musolf, Subleading logarithmic mass dependence in heavy meson form-factors, Phys. Lett. B 257 (1991) 409 [INSPIRE].

[57] K. Melnikov and T. van Ritbergen, The three loop on-shell renormalization of $Q C D$ and QED, Nucl. Phys. B 591 (2000) 515 [hep-ph/0005131] [INSPIRE].

[58] P. Marquard, A.V. Smirnov, V.A. Smirnov and M. Steinhauser, Four-loop wave function renormalization in QCD and QED, Phys. Rev. D 97 (2018) 054032 [arXiv:1801.08292] [INSPIRE].

[59] R. Brüser, A. Grozin, J.M. Henn and M. Stahlhofen, Matter dependence of the four-loop QCD cusp anomalous dimension: from small angles to all angles, JHEP 05 (2019) 186 [arXiv: 1902.05076] [INSPIRE].

[60] D.J. Broadhurst and A.G. Grozin, Operator product expansion in static quark effective field theory: Large perturbative correction, Phys. Lett. B 274 (1992) 421 [hep-ph/9908363] [INSPIRE].

[61] A. Czarnecki and K. Melnikov, Threshold expansion for heavy light systems and flavor off diagonal current current correlators, Phys. Rev. D 66 (2002) 011502 [hep-ph/0110028] [INSPIRE].

[62] M. Eidemuller and M. Jamin, QCD field strength correlator at the next-to-leading order, Phys. Lett. B 416 (1998) 415 [hep-ph/9709419] [INSPIRE].

[63] A.I. Vainshtein and V.I. Zakharov, Calculating Photonic Condensate in Perturbative QED, Phys. Lett. B 225 (1989) 415 [INSPIRE].

[64] K.G. Chetyrkin and A. Maier, Massless correlators of vector, scalar and tensor currents in position space at orders $\alpha_{s}^{3}$ and $\alpha_{s}^{4}$ : Explicit analytical results, Nucl. Phys. B 844 (2011) 266 [arXiv: 1010.1145] [INSPIRE].

[65] P.A. Baikov, K.G. Chetyrkin, J.H. Kühn and J. Rittinger, Vector Correlator in Massless $Q C D$ at $\operatorname{Order} \mathcal{O}\left(\alpha_{s}^{4}\right)$ and the QED $\beta$-function at Five Loop, JHEP 07 (2012) 017 [arXiv:1206.1284] [INSPIRE]. 